\title{
Epidemiological Impact of Universal Varicella Vaccination on Consecutive Emergency Department Visits for Varicella and Its Cost-Effectiveness Among Children
}

Hiroshi Yamaguchi ( $\nabla$ hiyamagu@med.kobe-u.ac.jp )

Kobe University

Kandai Nozu

Kobe University

Shinya Ishiko

Kobe University

Hiroaki Nagase

Kobe University

Takeshi Ninchoji

Kobe University

China Nagano

Kobe University

Hiroki Takeda

Kobe University

Ai Unzaki

Kobe Children's Primary Emergency Medical Center

Kazuto Ishibashi

Kobe Children's Primary Emergency Medical Center

Ichiro Morioka

Nihon University

Kazumoto lijima

Kobe University

Akihito Ishida

Kobe Children's Primary Emergency Medical Center

Research Article

Keywords: chicken pox, cost-effectiveness, emergency department, observational study, vaccine

Posted Date: December 30th, 2020

DOI: https://doi.org/10.21203/rs.3.rs-135408/v1

License: (1) This work is licensed under a Creative Commons Attribution 4.0 International License. Read Full License 


\section{Abstract}

Varicella is highly contagious and endemic worldwide. Previous studies reported a dramatic decline in varicella incidence and varicella-related deaths after implementing universal varicella vaccination (VarV). However, its impact and cost-effectiveness remain unknown in single emergency department (ED). We retrospectively reviewed the clinical database of consecutive patients younger than 16 years presenting to our primary ED between January 1 , 2011, and December 31, 2019. Of the 265,191 children presenting to our ED, 3,092 were clinically diagnosed with varicella. The annual number of varicella patients was approximately 500 before introduction of the universal twodose VarV in October 2014, and it decreased to approximately 200 in 2019. The number of varicella patients younger than 1 year (not eligible for vaccination) also decreased. Regarding the cost-effectiveness of VarV, approximately JPY1.5 million (US\$14,300) were saved annually by our center. However, our study showed a relatively large percentage of infected unvaccinated children presenting to our ED (59.0\%). After implementation of the universal VarV, infection was mainly observed in older children (i.e., the unvaccinated generation). In conclusion, our data showed excellent universal VarV effectiveness and cost-effectiveness in the ED. Additionally, our data suggest that the VarV should be administered to all eligible older patients.

\section{Introduction}

Varicella is caused by the varicella-zoster virus (VZV), which belongs to the alpha subfamily of Herpesviridae. VZV is highly contagious, transmitted through the air, and endemic to all countries. VZV infection is generally a mild illness consisting of moderate fever, mild cold symptoms, and a vesicular rash that develops scabs [1]. Some cases of varicella lead to serious complications such as encephalitis, pneumonia, and secondary bacterial infections; rarely, it results in death, especially for children younger than 1 year, immunocompromised patients, patients with impaired malignancies, and patients using immunosuppressive medications [1-4].

Previous studies reported a dramatic decline in the varicella incidence and varicella-related death after a universal varicella vaccination (VarV) program was implemented in many countries [5-9]. A single dose of the VarV decreases infections by approximately $80 \%$, and the two-dose VarV decreases infections by more than $95 \%[8,10,11]$. Furthermore, the number of hospitalizations caused by varicella has declined by approximately more than $95 \%$ [ 12 , 13]. Although the live attenuated Oka strain VarV was first developed in 1974 by Takahashi in Japan [14], the VarV was a voluntary inoculation in Japan until October 2014, when its administration was universally implemented. Before the implementation of the universal VarV in Japan, the number of individuals who received the VarV was estimated to be $40 \%$, and this low vaccination rate resulted in consistent outbreaks of varicella [15]. The two-dose schedule for VarV was adopted with an interval of at least 3 months for children 1 to 3 years of age, and a catch-up schedule was implemented for children 3 to 4 years of age during the first 6 months of its adoption. The descriptive epidemiology of varicella based on national surveillance data in Japan indicated a dramatic decline in varicella cases after the implementation of the universal VarV [9]. However, despite the effectiveness of the VarV, debates exist regarding its cost-effectiveness $[5,6,16,17]$. The cost of the VarV is significant. Lieu et al. reported that the annual cost of the VarV is approximately $\$ 162$ million in the United States [18]. Nevertheless, the universal VarV has strong benefits, such as reduced morbidity and mortality due to varicella and reduced costs associated with the time parents must utilize to care for their ill children [5-9]. However, its annual cost exceeded the annual savings cost of $\$ 80$ million in the United States [18]. There have been no reports of the effectiveness of the universal VarV and its cost-effectiveness for consecutive patients, especially those presenting to the emergency department (ED), in Japan. This study aimed to evaluate the effectiveness and cost-effectiveness of the universal VarV in Japan, especially for those who present to the ED. 


\section{Results}

\subsection{Patient demographics}

From 2011 to 2019, 265,191 pediatric patients visited our primary ED center. Of those, 3,092 patients were clinically diagnosed with varicella. The mean age was 4.8 years (SD, 3.0); $53.2 \%$ were boys $(n=1,646)$ and $46.8 \%$ were girls $(n$ $=1,446)$.

\subsection{Yearly changes in the total number of varicella patients}

The yearly change of the total number of varicella patients who visited our center is shown in Fig. 1A. The national universal immunization program of VarV was implemented in Oct 2014. Until 2014, the number of varicella patients at our center was approximately 500 annually. After the implementation of universal VarV program in 2014, the number of varicella patients dramatically decreased, and the number in 2015 was approximately half the number in 2014. This number constantly decreased until 2018. However, the number of patients slightly increased in 2019; therefore, there was a significant decrease in the number of varicella cases each year from 2011 to 2014 and from 2015 to 2019 ( $p=0.0159$; Mann-Whitney U-test).

The yearly changes in the mean age of varicella patients are shown in Fig. 1B. The mean age was approximately 4 years until 2014. After the implementation of the universal VarV, the mean age of varicella patients gradually increased to 7.0 years (SD, 3.2 years), which was significantly different $(4.5$ years [ $95 \% \mathrm{Cl}, 4.2-4.7]$ in 2014 vs 7.0 years [95\% $\mathrm{Cl}, 6.5-7.4]$ in 2019; $\mathrm{p}<0.0001$; Mann-Whitney U-test).

\subsection{Changes in the yearly number of varicella patients according to age}

The changes in the yearly number of varicella patients are shown in Fig. 2. After the implementation of the universal VarV in 2014, the number of patients younger than 6 years decreased. However, the number of patients 6 years or older tended to increase. Interestingly, the number of patients younger than 1 year who were not the eligible for the vaccination also decreased remarkably.

\subsection{Changes in the number of varicella patients according to birth year}

The changes in the number of varicella patients according to their birth year (from 2011 to 2019) are shown in Fig. 3. Although patients born in 2011, 2012, and 2013 are not part of the regular vaccination generation (voluntary inoculation), the number of varicella patients aged 4,3 , and 2 years clearly decreased since 2015 . Similarly, patients born in 2014 are part of the universal VarV generation (routine inoculation), and the number of 1-year-old varicella patients clearly decreased since 2014. The maximum number of varicella patients born before 2014 was more than 40 annually. However, children born after 2014 apparently benefited from the universal VarV because the maximum number decreased to less than approximately 20 annually.

\subsection{Annual estimated vaccination rate in Kobe City and annual vaccination history of varicella patients who visited our center}

The annual estimated VarV rate in Kobe City is shown in Table 1. This rate was calculated by dividing the number of births during the previous year by the number of vaccinations administered. The universal VarV program was started 
in Japan in October 2014. The estimated rate of receiving the first dose of VarV was more than $94.5 \%$ (lowest in 2017), and that of receiving the second dose was more than $85.0 \%$ (lowest in 2017, except for 2014, when the VarV was introduced). In 2014 and 2015, the VarV rate was extremely high, $99.8 \%$ and $103.5 \%$, respectively, probably because of the number of vaccinations administered to children 2 years or older who had not been vaccinated during the voluntary vaccination period or because of some children moving from another city to Kobe City. The annual history of the VarV for varicella patients at our center is shown in Table 2. Our center started to obtain the vaccination histories of patients in April 2016. Although the VarV history could be obtained, the number of doses (one or two) could not be obtained from our database. In 2016, 169 of 245 (69.0\%) patients had a history of VarV administration. In 2017, 197 of 214 (92.1\%) patients had a history of VarV administration. In 2018, 136 of 154 (88.3\%) patients had a history of VarV administration. In 2019, 177 of 207 (85.5\%) patients had a history of VarV administration. In $2016,64.5 \%$ of varicella patients did not have a history of VarV administration. In $2017,63.5 \%$ of varicella patients did not have a history of VarV administration. In 2018, 58.1\% of varicella patients did not have a history of VarV administration. In 2019, 53.7\% of varicella patients did not have a history of VarV administration. Double asterisks in Table 2 represents the generations of patients who were not part of universal VarV program. A total of 161 children in these generations were diagnosed with varicella at our center. Of these 161 children, 66 received at least one dose of the vaccination and 95 (59.0\%) were not vaccinated.

Table 1

VarV rate for the targeted age group in Kobe City estimated based on the number of births during the previous year

\begin{tabular}{|llllll|}
\hline & Births & \multicolumn{2}{l}{ VarV administered $(\mathrm{n})$} & \multicolumn{2}{l|}{ Vaccination rate (\%) } \\
\hline Year & in Kobe City $(\mathrm{n})$ & First dose & Second dose & First dose & Second dose \\
\hline 2013 & 12,437 & n.d. & n.d. & n.d. & n.d. \\
\hline 2014 & 12,218 & 12,413 & 5,334 & 99.8 & 42.9 \\
\hline 2015 & 12,140 & 12,650 & 12,753 & 103.5 & 104.4 \\
\hline 2016 & 12,124 & 11,985 & 10,760 & 98.7 & 88.6 \\
\hline 2017 & 11,565 & 11,455 & 10,305 & 94.5 & 91.1 \\
\hline 2018 & 10,711 & 11,196 & 10,532 & 96.8 & 93.0 \\
\hline 2019 & 10,468 & 10,181 & 9,957 & 95.1 & \\
\hline $\begin{array}{l}\text { n, number; } \text { n.d., no data } \\
\text { The vaccination rate was calculated by dividing the number of births during the previous year by the number of } \\
\text { vaccinations administered during the current year. In 2015, the VarV rate was more than 100\%. This was } \\
\text { probably due to some children moving from another city in 2015. }\end{array}$
\end{tabular}


Table 2

Annual vaccination history of varicella patients who visited our center

\begin{tabular}{|c|c|c|c|c|c|c|c|c|}
\hline \multirow[b]{3}{*}{$\begin{array}{l}\text { Age, } \\
\text { years }\end{array}$} & \multicolumn{2}{|c|}{$\begin{array}{l}2016 \text { (April-December) } \\
(\mathrm{n}=169 / 245)^{*}\end{array}$} & \multicolumn{2}{|c|}{$2017(\mathrm{n}=197 / 214)$} & \multicolumn{2}{|c|}{$2018(n=136 / 154)$} & \multicolumn{2}{|c|}{$2019(n=177 / 207)$} \\
\hline & \multicolumn{2}{|c|}{ Vaccination history } & \multicolumn{2}{|c|}{ Vaccination history } & \multicolumn{2}{|c|}{ Vaccination history } & \multicolumn{2}{|c|}{ Vaccination history } \\
\hline & $\begin{array}{l}\text { At least } \\
\text { one dose }\end{array}$ & none & $\begin{array}{l}\text { At least } \\
\text { one dose }\end{array}$ & None & $\begin{array}{l}\text { At least } \\
\text { one dose }\end{array}$ & None & $\begin{array}{l}\text { At least } \\
\text { one dose }\end{array}$ & None \\
\hline 0 & $0(0.0 \%)$ & $\begin{array}{l}10 \\
(100.0 \%)\end{array}$ & $0(0.0 \%)$ & $\begin{array}{l}11 \\
(100.0 \%)\end{array}$ & $0(0.0 \%)$ & $3(100.0 \%)$ & $0(0.0 \%)$ & $6(100.0 \%)$ \\
\hline 1 & $\begin{array}{l}5 \\
(33.3 \%) \star \star\end{array}$ & $\begin{array}{l}10 \\
(66.7 \%) \star \star\end{array}$ & $\begin{array}{l}5 \\
(33.3 \%)^{\star \star}\end{array}$ & $\begin{array}{l}10 \\
(66.7 \%) \star \star\end{array}$ & 6 & $\begin{array}{l}10 \\
(62.5 \%)^{\star \star}\end{array}$ & $\begin{array}{l}4 \\
(26.7 \%) \star \star\end{array}$ & $\begin{array}{l}11 \\
(73.3 \%) \star \star\end{array}$ \\
\hline 2 & $\begin{array}{l}3 \\
(42.9 \%) \star \star\end{array}$ & $\begin{array}{l}4 \\
(57.1 \%) \star \star\end{array}$ & $\begin{array}{l}11 \\
(57.9 \%)^{\star \star}\end{array}$ & $\begin{array}{l}8 \\
(42.1 \%) \star \star\end{array}$ & $\begin{array}{l}0 \\
(0.0 \%)^{\star \star}\end{array}$ & $\begin{array}{l}4 \\
(100.0 \%)^{\star \star}\end{array}$ & $\begin{array}{l}5 \\
(55.6 \%) * \star\end{array}$ & $\begin{array}{l}4 \\
(44.4 \%) \star \star\end{array}$ \\
\hline 3 & $4(50.0 \%)$ & $4(50.0 \%)$ & $\begin{array}{l}5 \\
(33.3 \%)^{\star \star}\end{array}$ & $\begin{array}{l}10 \\
(66.7 \%) \star *\end{array}$ & $\begin{array}{l}3 \\
(50.0 \%) \star *\end{array}$ & $\begin{array}{l}3 \\
(50.0 \%) * *\end{array}$ & $\begin{array}{l}0 \\
(0.0 \%) * *\end{array}$ & $\begin{array}{l}5 \\
(100.0 \%) * \star\end{array}$ \\
\hline 4 & $\begin{array}{l}10 \\
(50.0 \%)\end{array}$ & $\begin{array}{l}10 \\
(50.0 \%)\end{array}$ & $4(36.4 \%)$ & $7(63.6 \%)$ & $\begin{array}{l}6 \\
(60.0 \%) * \star\end{array}$ & $\begin{array}{l}4 \\
(40.0 \%)^{\star \star}\end{array}$ & $\begin{array}{l}2 \\
(20.0 \%) \star \star\end{array}$ & $\begin{array}{l}8 \\
(80.0 \%)^{\star \star}\end{array}$ \\
\hline 5 & $\begin{array}{l}13 \\
(43.3 \%)\end{array}$ & $\begin{array}{l}17 \\
(56.7 \%)\end{array}$ & $7(33.3 \%)$ & $\begin{array}{l}14 \\
(66.7 \%)\end{array}$ & $8(47.1 \%)$ & $9(52.9 \%)$ & $\begin{array}{l}11 \\
(73.3 \%)^{\star *}\end{array}$ & $\begin{array}{l}4 \\
(26.7 \%)^{\star \star}\end{array}$ \\
\hline 6 & $\begin{array}{l}10 \\
(50.0 \%)\end{array}$ & $\begin{array}{l}10 \\
(50.0 \%)\end{array}$ & $\begin{array}{l}10 \\
(55.6 \%)\end{array}$ & $8(44.4 \%)$ & $5(45.5 \%)$ & $6(54.5 \%)$ & $\begin{array}{l}13 \\
(50.0 \%)\end{array}$ & $13(50.0 \%)$ \\
\hline 7 & $2(11.8 \%)$ & $\begin{array}{l}15 \\
(88.2 \%)\end{array}$ & $\begin{array}{l}10 \\
(41.7 \%)\end{array}$ & $\begin{array}{l}14 \\
(58.3 \%)\end{array}$ & $6(60.0 \%)$ & $4(40.0 \%)$ & $8(47.1 \%)$ & $9(52.9 \%)$ \\
\hline 8 & $5(29.4 \%)$ & $\begin{array}{l}12 \\
(70.6 \%)\end{array}$ & $3(17.6 \%)$ & $\begin{array}{l}14 \\
(82.4 \%)\end{array}$ & $8(42.1 \%)$ & $11(57.9 \%)$ & $\begin{array}{l}11 \\
(55.0 \%)\end{array}$ & $9(45.0 \%)$ \\
\hline 9 & $1(16.7 \%)$ & $5(83.3 \%)$ & $4(26.7 \%)$ & $\begin{array}{l}11 \\
(73.3 \%)\end{array}$ & $4(25.0 \%)$ & $12(75.0 \%)$ & $6(42.9 \%)$ & $8(57.1 \%)$ \\
\hline 10 & $1(33.3 \%)$ & $2(66.7 \%)$ & $5(33.3 \%)$ & $\begin{array}{l}10 \\
(66.7 \%)\end{array}$ & $2(22.2 \%)$ & $7(77.8 \%)$ & $\begin{array}{l}10 \\
(58.8 \%)\end{array}$ & $7(41.2 \%)$ \\
\hline 11 & $3(37.5 \%)$ & $5(62.5 \%)$ & $5(71.4 \%)$ & $2(28.6 \%)$ & $3(75.0 \%)$ & $1(25.0 \%)$ & $3(42.9 \%)$ & $4(57.1 \%)$ \\
\hline 12 & $1(25.0 \%)$ & $3(75.0 \%)$ & $1(16.7 \%)$ & $5(83.3 \%)$ & $4(44.4 \%)$ & $5(55.6 \%)$ & $7(63.6 \%)$ & $4(36.4 \%)$ \\
\hline 13 & $0(0.0 \%)$ & $0(0.0 \%)$ & $0(0.0 \%)$ & $0(0.0 \%)$ & $\begin{array}{l}1 \\
(100.0 \%)\end{array}$ & $0(0.0 \%)$ & $0(0.0 \%)$ & $1(100.0 \%)$ \\
\hline 14 & $2(50.0 \%)$ & $2(50.0 \%)$ & $2(66.7 \%)$ & $1(33.3 \%)$ & $\begin{array}{l}1 \\
(100.0 \%)\end{array}$ & $0(0.0 \%)$ & $2(66.7 \%)$ & $1(33.3 \%)$ \\
\hline 15 & $0(0.0 \%)$ & $0(0.0 \%)$ & $0(0.0 \%)$ & $0(0.0 \%)$ & $0(0.0 \%)$ & $0(0.0 \%)$ & $0(0.0 \%)$ & $1(100.0 \%)$ \\
\hline Total & 60 & $\begin{array}{l}109 \\
(64.5 \%)\end{array}$ & 72 & $\begin{array}{l}125 \\
(63.5 \%)\end{array}$ & 57 & $79(58.1 \%)$ & 82 & $95(53.7 \%)$ \\
\hline \multicolumn{9}{|c|}{ Data are presented as number (\%). The gray areas represent the regular vaccination generations. } \\
\hline $\begin{array}{ll}* V \\
p a\end{array}$ & inn hint & onoilah & & & & & & \\
\hline
\end{tabular}




\subsection{Cost-effectiveness of the VarV in relation to ED visits}

The medical fee for visiting our center for varicella depends on age. However, the average fee per patient at our hospital is approximately JPY15,000 (US\$143) for the medical examination, acyclovir, and ointment. The cost of one dose of VarV is approximately JPY 5,000 in Japan; thus, the cost of two doses is approximately JPY10,000. Our data showed that the annual number of varicella patients was approximately 500 before 2014; however, after 2014, the annual number was approximately 200. Therefore, an approximate calculation (300 × [JPY15,000 minus JPY10,000]) indicated that JPY1.5 million (US\$14,300) was saved annually by our center. Additionally, considering the risk of infection to other people in hospitals, schools, and communities, the loss associated with being unable to attend school, and the loss associated with parents having to miss work to care for their children, the benefits of universal VarV are expected to outweigh the cost.

We conducted a retrospective observational study of the effectiveness and cost-effectiveness of the universal VarV by analyzing consecutive patients who visited our primary ED center. We showed that after the implementation of the universal VarV in 2014, the number of children with varicella who presented to our ED dramatically decreased and JPY1.5 million (US\$14,300) was saved annually by our center alone.

Currently, 33 countries and regions have introduced the universal VarV since 2014 [19]. The effectiveness of the VarV was proven in many countries, and a dramatic decline in the incidence of varicella has been reported [5-8, 20]. Although one dose of the VarV is highly protective against severe varicella, two doses of the VarV further improved the rates of protection from varicella $[5,8]$. In the United States, the one-dose VarV program was implemented in 1996, and the two-dose VarV program was started in 2007. An $87 \%$ reduction in varicella deaths occurred during 2008-2011 compared with the pre-vaccine period (1990-1994) [21]. A meta-analysis of the effectiveness of the VarV revealed the superiority of the two-dose VarV (estimated at $81 \%$ for one dose and $92 \%$ for two doses) [8]. Immediately after the introduction of the universal VarV in Japan, Hattori et al. reported the effectiveness of the VarV (Oka/Biken) using a case-control study; they showed that the VarV effectiveness rates were $76.9 \%$ and $94.7 \%$ for one dose and two doses, respectively [10]. These data showed the superiority of the routine two-dose VarV. The data obtained in the ED setting also showed a dramatic decline in the number of varicella patients after implementation of the universal two-dose VarV. Additionally, we showed that a decline occurred among children younger than 1 year who were not yet eligible for the vaccination. These effects of the VarV are considered important from the viewpoint of not infecting other patients in the ED.

Cost-effectiveness is an important issue that should be addressed. In the present study, we reported that the average fee per patient at our center is approximately JPY15,000 (US\$143), and that JPY1.5 million (US\$14,300) was saved annually by our center. Before the universal VarV program was initiated, according to the report by the Varicella Vaccination Working Team, Infectious Diseases Subcommittee, and Health Science Council, it was estimated that the universal two-dose VarV could be expected to reduce costs by approximately JPY36.23 billion (US\$348 million) per year in Japan ( http://www.mhlw.go.jp/stf/shingi/2r98520000014wdd-att/2r98520000016rqn.pdf ).

A sensitivity analysis of the cost-effectiveness of VarV showed apparent cost-savings from the perspectives of society and the health system in European countries [6]. In the United States, before the availability of the VarV, 4 million cases of varicella occurred each year; of these, more than approximately 10,000 required hospitalization and more than 100 resulted in death [22]. Previous studies estimated that the universal VarV during childhood is beneficial, but there is a debate regarding whether it is more cost-effective to administer one dose or two doses [23, 24]. Thus, several previous studies as well as our data revealed the cost-effectiveness of two-dose VarV. 
Our data showed that the current VarV program in Kobe City is not sufficiently widespread. The present study clearly showed that the age of varicella patients who presented to our ED has increased since the implementation of the universal two-dose VarV. Previous studies also reported that the occurrence of varicella has shifted to older children $[9,20]$. Qin et al. reported the shift of varicella to older children after the implementation of one-dose VarV in Lu'an, China [25]. They mentioned that this was attributable to the one-dose voluntary vaccination policy. The World Health Organization position is that the VarV administration rate, which has been less than $80 \%$, is expected to shift varicella to older children [26]. However, in the present study, although the two-dose VarV was implemented, approximately more than $95 \%$ of children have received a single dose and approximately more than $85 \%$ of children have received two doses; therefore, a shift of varicella to older children has been observed. Based on national surveillance data from 2000 to 2017, Morino et al. also reported the shift of varicella to older children in Japan after the introduction of the VarV in the routine vaccination program [9]. Although they showed that the incidence of varicella in 1- to 4-year-olds declined to $34.8 \%$ in 2017 , the incidence in 5- to 9-year-olds relatively increased from $20.5 \%$ during $2000-2011$ to $51.6 \%$ in 2017 , even though the number of cases occurring in 5 - to 9 -year-olds had decreased. In the present study, it was found that the number of varicella cases in children older than 5 years increased in 2019. Therefore, to prevent any potential shift to older children, it may be necessary to introduce the VarV not only to the non-vaccinated target age group but also to unvaccinated and single-vaccinated children. In 2012, before the universal VarV program started, the Japan Pediatric Society recommended the VarV and stated that the two-dose VarV was required to ensure its preventive effects. However, our study clearly showed that these recommendations were insufficiently supported based on the increased number of varicella patients 1-2 years of age between 2012 and 2013. Therefore, national support is needed to increase the VarV administration rate and decrease the number varicella cases. In the present study, the number of varicella patients younger than 1 year (who were not the target age for VarV) presenting to the ED also declined. This result suggests the herd protection effect of the VarV, and the same effect was observed in other countries [27]. Our data revealed that many unvaccinated children were diagnosed with varicella at our center (59.0\%). Because the proportion of unvaccinated individuals is $5 \%$ in Kobe City, 1,805 children in Kobe City are estimated to be unvaccinated. If that 1,805 were reduced to 66 , then the reduction rate would be approximately $96.3 \%$. Although that reduction rate seems high, our data showed that the number of unvaccinated patients with varicella presenting to our center was relatively high (53.7\%-64.5\%).

Therefore, the VarV administration rate for children in the current target age group must be increased to reduce the number of varicella patients.

Our study had many strengths. Because a relatively large number of consecutive patients were included, we were able to analyze the effectiveness of the VarV for each age in detail at the ED. The secondary effect of the vaccine for those younger than 1 year who are not eligible for the VarV could be proven more strongly because the total number of consecutive patients with VZV infection was large. Our observational study period was relatively long ( 9 years). Finally, we obtained the VarV history of most patients. However, our study also had some limitations. First, this study was conducted at a single center. Therefore, it is unknown whether our results can be applied to other areas. Second, detailed patient backgrounds were unavailable in our database. Third, there was a possible misclassification bias during the study of confirmed cases because a clinical sample could not be extracted and the diagnosis was clinically determined. Regardless of these limitations, our data clearly showed the excellent effectiveness and costeffectiveness of the VarV in the ED setting.

In conclusion, our data showed a dramatic decline in the number of varicella patients who presented to the ED after the implementation of the universal two-dose VarV. These data also showed that the benefits of the VarV outweigh its cost. Currently, the VarV program in Japan is insufficiently implemented. Our data suggest that we need to 
increase the VarV administration rate for children in the target age group, and that public assistance is required to expand VarV administration to older children.

\section{Material And Methods \\ 3.1. Study design and patients}

This retrospective, clinical observational time-series study was conducted under the approval of the Ethics Committee of Kobe Children's Primary Emergency Medical Center. Informed consent was waived because of the retrospective nature of the study and accessibility to the public using our website. To determine the effectiveness and cost-effectiveness of the universal VarV, we reviewed the clinical database of consecutive patients younger than 16 years who presented to the ED of Kobe Children's Primary Emergency Medical Center, which is the largest nighttime emergency medical center in Kobe City in Japan, between January 1, 2011 and December 31, 2019. Kobe City is located in the southern part of the main island of Japan; there are approximately 1,500,000 residents, of whom 200,000 are children younger than 16 years. A total of 265,191 children visited the center during the study period and were included in the present study.

\subsection{VarV distribution and births in Kobe City}

Data regarding the VarV distribution in Kobe City were obtained from the Kobe City General Education Center, and the number of annual births in Kobe City was obtained from the Kobe City website (http://www.city.kobe.Ig.jp/).

\subsection{Statistical Analysis}

The results are expressed as the number (\%) or mean (standard deviation [SD] or 95\% confidential interval [CI]). The Mann-Whitney U-test was used as appropriate to analyze the data. All analyses were performed using GraphPad Prism 5.0 (GraphPad Software, San Diego, CA). P < 0.05 was considered statistically significant.

\section{Declarations}

\section{Acknowledgments}

The authors are grateful to the physicians at the Kobe Children's Primary Emergency Medical Center for treating varicella patients.

\section{Author contributions}

H.Y.: Conceptualization, methodology, formal analysis, investigation, and writing the original draft. K.N.: Conceptualization, methodology, formal analysis, investigation, writing the original draft, and supervision. S.I.: Conceptualization, methodology, formal analysis, and investigation. H.N.: Writing (review and editing) and supervision. T.N.: Writing (review and editing) and supervision. C.N.: Writing (review and editing) and supervision. H.T.: Writing (review and editing) and supervision. A.U.: Writing (review and editing) and supervision. K. I.: Writing (review and editing) and supervision. I.M.: Writing (review and editing) and supervision. K.I.: Writing (review and editing) and supervision. A.I.: Resources, writing (review and editing), and supervision. All authors have read and approved the final manuscript. 


\section{Funding}

This research did not receive any specific grant from funding agencies in the public, commercial, or not-for-profit sectors.

\section{Conflicts of interest}

The authors declare no conflicts of interest.

\section{References}

1. Arvin, A. M. Varicella-zoster virus. Clin Microbiol Rev. 9, 361-381 https://doi.org/10.1128/CMR.9.3.361381.1996 (1996).

2. Rodriguez-Santana, Y., Sanchez-Almeida, E., Garcia-Vera, C., Garcia-Ventura, M. \& Martinez-Espligares, L. Epidemiological and clinical characteristics and the approach to infant chickenpox in primary care. Eur $J$ Pediatr. 178, 641-648 https://doi.org/10.1007/s00431-019-03332-9 (2019).

3. Neyro, S. E. et al. Clinical and epidemiological impact of varicella infection in children prior to the introduction of the varicella vaccine in the national immunization schedule of Argentina. Arch Argent Pediatr. 117, 12-18 https://doi.org/10.5546/aap.2019.eng.12 (2019).

4. Yamaguchi, H. et al. Streptococcus pulmonary empyema after varicella infection in a serologically immunocompetent boy. Pediatr Int. 56, 618-621 https://doi.org/10.1111/ped.12300 (2014).

5. Varela, F. H., Pinto, L. A. \& Scotta, M. C. Global impact of varicella vaccination programs. Hum Vaccin Immunother. 15, 645-657 https://doi.org/10.1080/21645515.2018.1546525 (2019).

6. Spoulou, V. et al. Implementing Universal Varicella Vaccination in Europe: The Path Forward. Pediatr Infect Dis J. 38, 181-188 https://doi.org/10.1097/INF.0000000000002233 (2019).

7. Jung, J. et al. Epidemiological Impact of the Korean National Immunization Program on Varicella Incidence. $J$ Korean Med Sci. 34, e53 https://doi.org/10.3346/jkms.2019.34.e53 (2019).

8. Yin, M., Xu, X., Liang, Y. \& Ni, J. Effectiveness, immunogenicity and safety of one vs. two-dose varicella vaccination: a meta-analysis. Expert Rev Vaccines. 17, 351-362 https://doi.org/10.1080/14760584.2018.1433999 (2018).

9. Morino, S. et al. Descriptive epidemiology of varicella based on national surveillance data before and after the introduction of routine varicella vaccination with two doses in Japan, 2000-2017. Vaccine. 36, 5977-5982 https://doi.org/10.1016/j.vaccine.2018.08.048 (2018).

10. Hattori, F. et al. Evaluating the effectiveness of the universal immunization program against varicella in Japanese children. Vaccine. 35, 4936-4941 https://doi.org/10.1016/j.vaccine.2017.07.090 (2017).

11. Latasa, P. et al. Effectiveness and impact of a single-dose vaccine against chickenpox in the community of Madrid between 2001 and 2015. Hum Vaccin Immunother. 14, 2274-2280 https://doi.org/10.1080/21645515.2018.1475813 (2018).

12. Leung, J. \& Marin, M. Update on trends in varicella mortality during the varicella vaccine era-United States, 1990-2016. Hum Vaccin Immunother. 14, 2460-2463 https://doi.org/10.1080/21645515.2018.1480283 (2018). 
13. Streng, A., Grote, V., Rack-Hoch, A. \& Liese, J. G. Decline of Neurologic Varicella Complications in Children During the First Seven Years After Introduction of Universal Varicella Vaccination in Germany, 2005-2011. Pediatr Infect Dis J. 36, 79-86 https://doi.org/10.1097/INF.0000000000001356 (2017).

14. Takahashi, M., Otsuka, T., Okuno, Y., Asano, Y. \& Yazaki, T. Live vaccine used to prevent the spread of varicella in children in hospital. Lancet. 2, 1288-1290 https://doi.org/10.1016/s0140-6736(74)90144-5 (1974).

15. Yoshikawa, T., Kawamura, Y. \& Ohashi, M. Universal varicella vaccine immunization in Japan. Vaccine. 34, 1965-1970 https://doi.org/10.1016/j.vaccine.2016.02.058 (2016).

16. Wysocki, J. et al. Varicella in Poland: economic burden in children 1-12 years of age in Poland, 2010-2015. BMC Public Health. 18, 410 https://doi.org/10.1186/s12889-018-5298-8 (2018).

17. Melegaro, A. et al. The impact of demographic changes, exogenous boosting and new vaccination policies on varicella and herpes zoster in Italy: a modelling and cost-effectiveness study. BMC Med. 16, 117 https://doi.org/10.1186/s12916-018-1094-7 (2018).

18. Lieu, T. A. et al. Cost-effectiveness of a routine varicella vaccination program for US children. JAMA. 271, 375381 (1994).

19. Wutzler, P. et al. Varicella vaccination - the global experience. Expert Rev Vaccines. 16, 833-843 https://doi.org/10.1080/14760584.2017.1343669 (2017).

20. Choi, J. K. et al. Trends in varicella and herpes zoster epidemiology before and after the implementation of universal one-dose varicella vaccination over one decade in South Korea, 2003-2015. Hum Vaccin Immunother. 15, 2554-2560 https://doi.org/10.1080/21645515.2019.1603985 (2019).

21. Leung, J., Bialek, S. R. \& Marin, M. Trends in varicella mortality in the United States: Data from vital statistics and the national surveillance system. Hum Vaccin Immunother. 11, 662-668

https://doi.org/10.1080/21645515.2015.1008880 (2015).

22. Shaw, J. \& Gershon, A. A. Varicella Virus Vaccination in the United States. Viral Immunol. 31, 96-103 https://doi.org/10.1089/vim.2017.0136 (2018).

23. Papaloukas, O., Giannouli, G. \& Papaevangelou, V. Successes and challenges in varicella vaccine. Ther $A d v$ Vaccines. 2, 39-55 https://doi.org/10.1177/2051013613515621 (2014).

24. Zhou, F., Harpaz, R., Jumaan, A. O., Winston, C. A. \& Shefer, A. Impact of varicella vaccination on health care utilization. JAMA. 294, 797-802 https://doi.org/10.1001/jama.294.7.797 (2005).

25. Qin, W. et al. The impact of long-term moderate level of vaccination coverage for epidemiology of varicella in Lu'an, China: should we change immunisation strategy now?. Epidemiol Infect. 148, e74 https://doi.org/10.1017/S0950268820000667 (2020).

26. Varicella and herpes zoster vaccines: WHO position paper, June 2014. Wkly Epidemiol Rec.89,265-87(2014).

27. Streng, A., Grote, V., Carr, D., Hagemann, C. \& Liese, J. G. Varicella routine vaccination and the effects on varicella epidemiology - results from the Bavarian Varicella Surveillance Project (BaVariPro), 2006-2011. BMC Infect Dis. 13, 303 https://doi.org/10.1186/1471-2334-13-303 (2013).

\section{Figures}



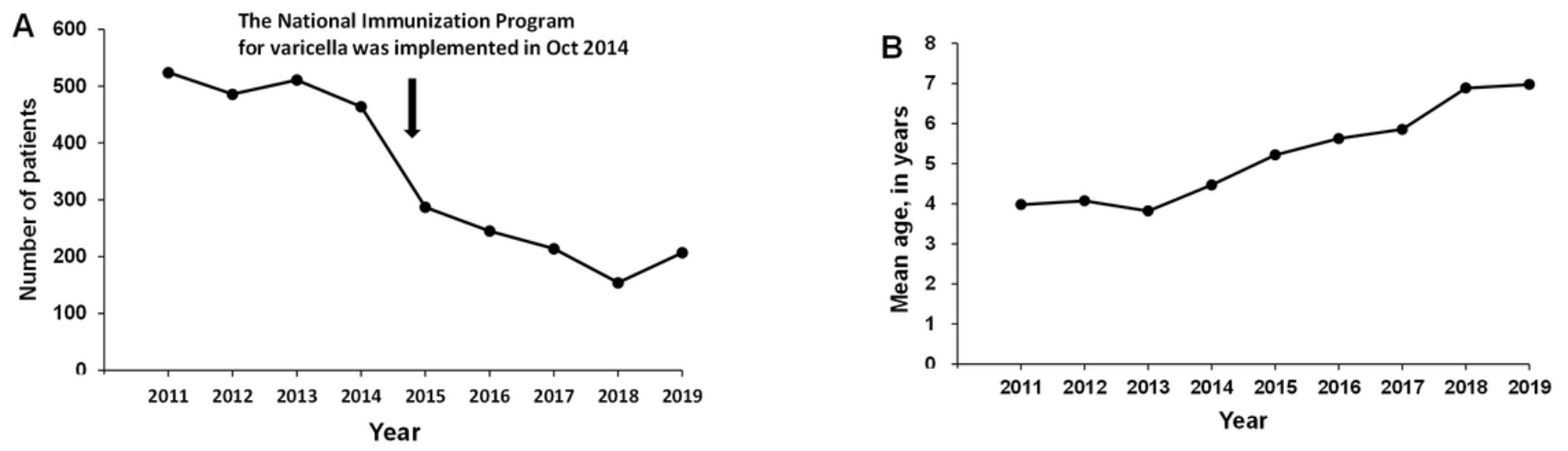

Figure 1

Yearly changes in the number of varicella patients $(A)$ and ages of patients with varicella (B). Data are presented as means.

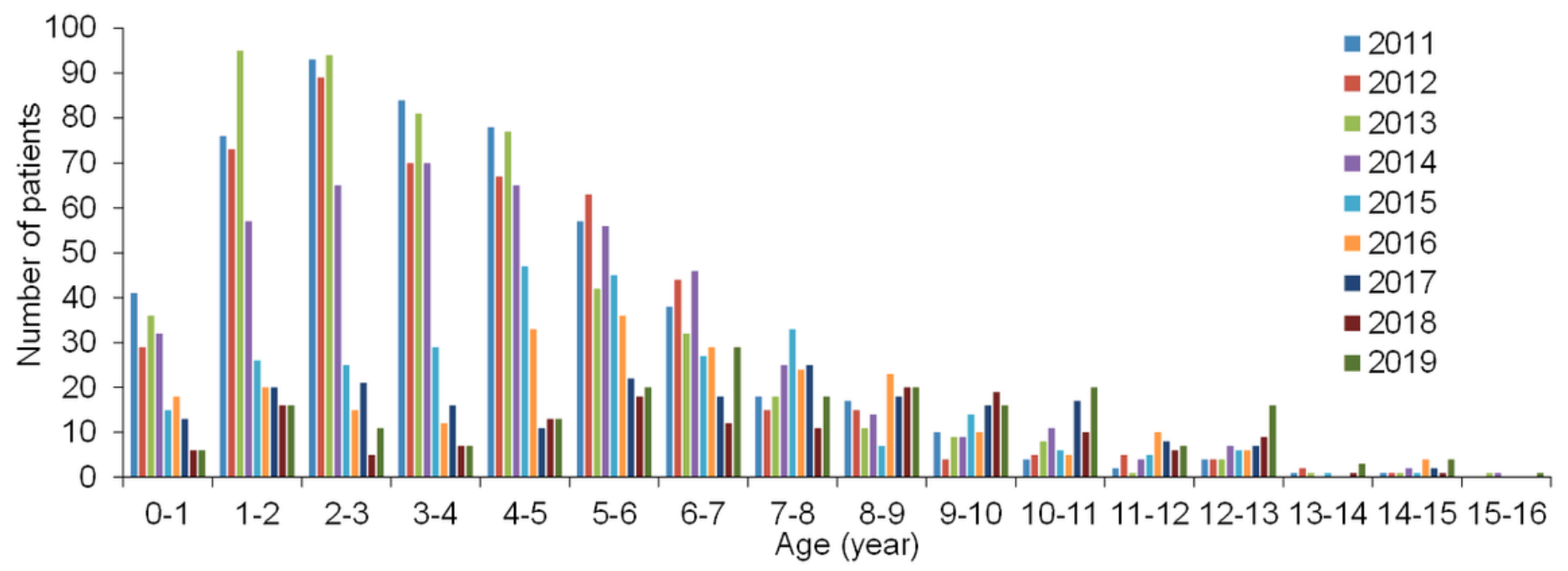

Figure 2

Changes in the yearly number of patients with varicella 


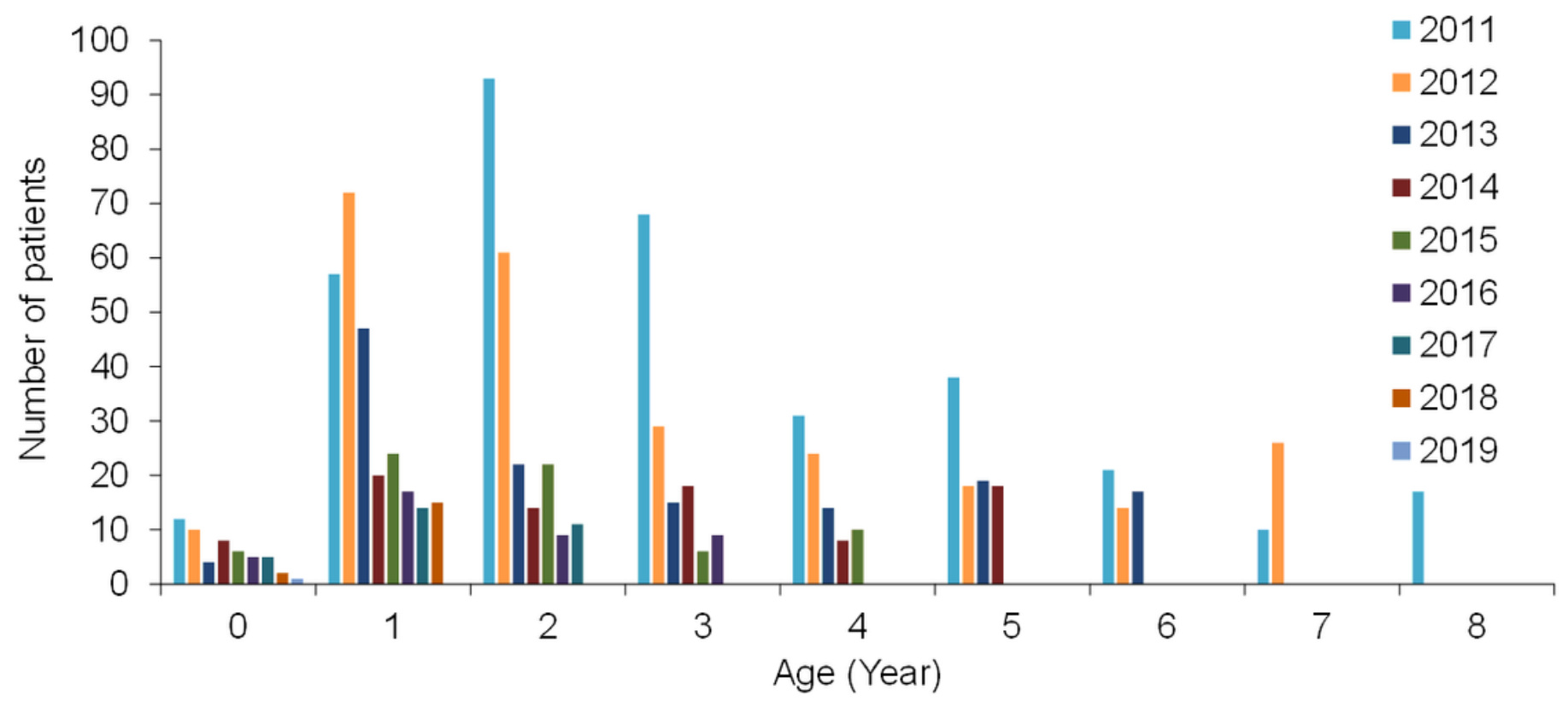

Figure 3

Changes in the number of varicella patients according to birth year from 2011 to 2019 